\title{
STEADY VORTEX FLOWS OBTAINED FROM AN INVERSE PROBLEM
}

\author{
B. EMAMiZADEh AND F. BAHRAMI
}

In this paper we prove the existence of solutions to an inverse semilinear elliptic partial differential equation. Physically, solutions represent stream functions of steady planar flows with bounded vortices. The kinetic energy functional is maximised over the set of rearrangements of a given function.

\section{INTRODUCTION}

In this paper we prove the existence of a solution to the following inverse semilinear partial differential equation:

$$
\left\{\begin{array}{c}
-\Delta u=\phi(u) \text { in } \Omega \\
u=0 \text { on } \partial \Omega \\
-\Delta u \in \mathcal{F}
\end{array}\right.
$$

where $\mathcal{F}$ is the set of rearrangements of a given function (see below). The nonlinearity $\phi$ is not known a priori, hence the problem is classified as an inverse problem. The domain $\Omega$ is an unbounded subset of the first quadrant $\Pi_{+}$.

In order to prove the existence of solutions to the above problem we use variational methods. However, the unboundedness of $\Omega$ causes lack of compactness, hence the direct method of the calculus of variations will not be applicable in our variational analysis. We shall apply the method suggested by Benjamin [3] complemented with a standard rescaling $([11,12])$ in order to compensate for the lack of compactness.

Physically, if $u$ represents the stream function of an incompressible planar flow, then $-\Delta u$ can be interpreted as the vorticity function of the fluid. Thus, from (1) we infer, formally that,

$$
[u,-\Delta u]=0
$$

in $\Omega$, where $[\cdot, \cdot]$ denotes the Jacobian. Equation (2) is the stream-vorticity representation of the two dimensional steady Euler equation. Therefore solving problem (1) proves the

Received 19th November, 2001

this research was in part supported by a grant from IPM.

Copyright Clearance Centre, Inc. Serial-fee code: 0004-9727/02 \$A2.00+0.00. 
existence of a two dimensional steady flow which is tangential to $\partial \Omega$. Condition $-\Delta u \in \mathcal{F}$ states that all possible configurations of the vorticity function are known.

Similar work has been done by Badiani [2], where the author makes extensive use of the symmetry of the domain (upper half-plane), contrary to the situation to be considered here. The reader may refer to earlier works of the first author $[5,6,7]$, which are the main references for the present work.

\section{NOTATION, DEFINITIONS, AND STATEMENT OF THE MAIN RESULT}

We denote by $p$ an arbitrary fixed number in $(2, \infty)$. For any number $r \geqslant 1, r^{*}$ denotes the conjugate exponent, $1 / r+1 / r^{*}=1$. For any measurable set $E \subseteq \mathbb{R}^{2}$, we denote its Lebesgue measure by $|E|$. By $B_{\xi}(x)$ we denote the ball centred at $x \in \mathbb{R}^{2}$ with radius $\xi$; if the centre is the origin we write $B_{\xi}$.

Let $D$ be an open, bounded, simply connected set containing the origin and assume $\bar{D} \subset B_{1}$. Let $\Pi_{+}$denote the open first quadrant; let $\Omega=\Pi_{+} \backslash \bar{D}$ such that $\partial \Omega \in C^{2}$. For $c>0$ we set

$$
\Omega_{c}=\left\{x \in \Pi_{+} \mid c^{1 / 2} x \in \Omega\right\}
$$

and $\Omega_{c, \xi}=\Omega_{c} \cap B_{\xi}$.

By $G$, with any subscripts, we denote the Green's function for $-\Delta$ with homogeneous Dirichlet boundary conditions in some domain; in particular, $G_{+}, G$ and $G_{1}$ denote the Green's functions in $\Pi_{+}, \Omega$ and $\Pi_{+} \backslash \bar{B}_{1}$, respectively. It is well known that

$$
G_{+}(x, y)=\frac{1}{2 \pi} \log \frac{|x-\bar{y}||x-\underline{y}|}{|x-y||x-\underline{y}|}, x, y \in \Pi_{+}, x \neq y .
$$

Furthermore, it is easy to see that

$$
G_{1}(x, y):=G_{+}(x, y)-\frac{1}{2 \pi} \log \frac{|y|\left|x-\overline{y^{*}}\right||y| \mid x-\underline{y^{*} \mid}}{|y|\left|x-y^{*}\right||y| \mid x-\underline{\overline{y^{*}} \mid}}, x, y \in \Pi_{+} \backslash B_{1}, x \neq y .
$$

Here the overline and the underline mean reflection about the $x_{1}$-axis and $x_{2}$-axis respectively, and * indicates inversion with respect to the unit circle.

REMARK. Let us point out that $G_{1}$ can be written more simply but we prefer this representation since it is more convenient later.

Note that

$$
G_{c}(x, y)=G\left(c^{1 / 2} x, c^{1 / 2} y\right), \quad x, y \in \Omega_{c}, x \neq y .
$$

By applying the Maximum Principle we obtain

$$
G_{1}(x, y) \leqslant G(x, y) \leqslant G_{+}(x, y)
$$

where each inequality holds in the positive domain. 
For a measurable function $\zeta$ and $x \in \mathbb{R}^{2}$ we define

$$
\begin{aligned}
K_{+} \zeta(x) & =\int_{\Pi_{+}} G_{+}(x, y) \zeta(y) d y \\
K \zeta(x) & =\int_{\Omega} G(x, y) \zeta(y) d y \\
K_{c} \zeta(x) & =\int_{\Omega_{c}} G_{c}(x, y) \zeta(y) d y,
\end{aligned}
$$

whenever the integrals exist.

We let $\eta \in C^{2}(\Omega) \cap C^{1}(\bar{\Omega})$ be a function such that

$$
\left\{\begin{array}{rlrl}
\Delta \eta & =0 \text { in } \Omega \\
\eta & =0 \text { on } \partial \Omega \\
\eta & =x_{1} x_{2}+O\left(|x|^{-2}\right), & & \text { as }|x| \rightarrow \infty \\
\nabla \eta & =\left(x_{2}, x_{1}\right)+O\left(|x|^{-3}\right), & & \text { as }|x| \rightarrow \infty
\end{array}\right.
$$

REMARK. The existence of $\eta$ can be proved using a standard limiting process; in addition one can use the maximum principle to show that

$$
x_{1} x_{2}-\frac{x_{1} x_{2}}{|x|^{4}} \leqslant \eta \leqslant x_{1} x_{2}, x \in \Omega .
$$

Next, for a measurable function $\zeta$ on $\Omega$ we define

$$
\begin{aligned}
& \Psi(\zeta)=\frac{1}{2} \int_{\Omega} \zeta K \zeta \\
& \mathfrak{F}(\zeta)=\int_{\Omega} \eta \zeta,
\end{aligned}
$$

whenever the integrals exist. Now fix $\lambda>0$. For a measurable function $\zeta$ on $\Omega$, we define

$$
\Psi_{\lambda}(\zeta)=\Psi(\zeta)-\lambda \mathfrak{F}(\zeta)
$$

Let us fix $\zeta_{0} \in L^{p}(\Omega)$ which is a non-negative, non-trivial function with compact support and assume $\left|\operatorname{supp}\left(\zeta_{0}\right)\right|=\pi a^{2}$, for some $a>0$. Moreover, we suppose that $\left\|\zeta_{0}\right\|_{1}=1$. By $\mathcal{F}$ we denote the set of rearrangements of $\zeta_{0}$ on $\Omega$ which have compact support. Let us recall that $\zeta$ is a rearrangement of $\zeta_{0}$ whenever

$$
|\{x \in \Omega \mid \zeta(x) \geqslant \alpha\}|=\left|\left\{x \in \Omega \mid \zeta_{0}(x) \geqslant \alpha\right\}\right|,
$$

for every $\alpha \in \mathbb{R}$. We now define the variational problem

$$
P_{\lambda}: \sup _{\zeta \in \mathcal{F}} \Psi_{\lambda}(\zeta)
$$


and the corresponding solution set is denoted $\Sigma_{\lambda}$. In order to introduce the second variational problem which is a rescaled version of $P_{\lambda}$ we proceed as follows. Let $c>0$ and let $\zeta$ be a measurable function on $\Omega$. Then we define

$$
\mathcal{C}(\zeta)(x)=c \zeta\left(c^{1 / 2} x\right), \quad x \in \Omega_{c} .
$$

The mapping $\mathcal{C}$ as define in (4) takes measurable functions on $\Omega$ to measurable functions on $\Omega_{c}$. By $\mathcal{F}_{c}$ we denote the set of all rearrangements of $\mathcal{C}\left(\zeta_{0}\right)$ on $\Omega_{c}$ with compact support.

For a measurable function $\zeta$ on $\Omega_{c}, c>0$, we define

$$
\widehat{\Psi}_{c}(\zeta):=\frac{1}{2} \int_{\Omega_{c}} \zeta K_{c} \zeta-\int_{\Omega_{c}} \eta_{c} \zeta,
$$

where $\eta_{c}(x):=c^{-1} \eta\left(c^{1 / 2} x\right)$, whenever the integrals exist. Now we define the rescaled variational problem. Fix $c>0$, then

$$
\widehat{P}_{c}: \sup _{\zeta \in \mathcal{F}_{c}} \widehat{\Psi}_{c}(\zeta),
$$

and $\widehat{\Sigma}_{c}$ denotes the corresponding solution set. Furthermore, for $\xi>1$ we define

$$
\widehat{P}_{c, \xi}: \sup _{\zeta \in \mathcal{F}_{c, \xi}} \widehat{\Psi}_{c}(\zeta)
$$

where $\mathcal{F}_{c, \xi}$ is the subset of $\mathcal{F}_{c}$ comprising functions vanishing outside $\Omega_{c, \xi}$. Let us point out that in order to ensure $\mathcal{F}_{c, \xi} \neq \emptyset$ it is sufficient to assume that

$$
\xi>\left(\frac{c^{2}+4 a^{2}}{c}\right)^{1 / 2}
$$

The solution set for $P_{c, \xi}$ is denoted by $\Sigma_{c, \xi}$.

The main result of this paper is the following.

THEOREM. There exists $\lambda_{0}>0$ such thatof $\lambda \in\left(0, \lambda_{0}\right), P_{\lambda}$ has a solution. If $\widehat{\zeta}_{\lambda}$ is a solution and $\psi_{\lambda}:=K \widehat{\zeta}_{\lambda}$, then $\psi_{\lambda}$ satisfies the following semilinear elliptic partial differential equation

$$
-\Delta \psi_{\lambda}=\phi_{\lambda} \circ\left(\psi_{\lambda}-\lambda \eta\right), \text { almost everywhere in } \Omega
$$

where $\phi_{\lambda}$ is an increasing function, unknown a priori.

\section{Preliminaries}

In this section we derive some properties of the operator $K$ which will enable us to use a result of Burton [4, Lemma 5], crucial in our analysis. Let us begin by noting that

$$
\begin{aligned}
G(x, y) & =\frac{1}{2 \pi} \log \frac{1}{|x-y|}-h(x, y) \\
G_{+}(x, y) & =\frac{1}{2 \pi} \log \frac{1}{|x-y|}-h_{+}(x, y) \\
G_{1}(x, y) & =\frac{1}{2 \pi} \log \frac{1}{|x-y|}-h_{1}(x, y),
\end{aligned}
$$


where $h, h_{+}$and $h_{1}$ are harmonic functions, for fixed $y$, in their respective domains. In particular, we have

$$
\begin{aligned}
& h_{+}(x, y)=\frac{1}{2 \pi} \log \frac{|x-\bar{y}|}{|x-\bar{y}||x-\underline{y}|} \\
& h_{1}(x, y)=h_{+}(x, y)+\frac{1}{2 \pi} \log \frac{|y|\left|x-\overline{y^{*}}\right||y| \mid x-\underline{\underline{y^{*}} \mid}}{|y|\left|x-\bar{y}^{*}\right||y| \mid x-\underline{\overline{y^{*}} \mid}}
\end{aligned}
$$

Note that

$$
h_{+}(x, y) \leqslant h(x, y) \leqslant h_{1}(x, y) \text {, }
$$

where the inequalities are understood to hold in the positive domains. Next we set $\widehat{h}=h-h_{+}$and $\widehat{h}_{1}=h_{1}-h_{+}$. Then from (8) we infer $0 \leqslant \widehat{h} \leqslant \widehat{h}_{1}$. Let us now point out that

$$
\widehat{h}_{1}(x, y)=\frac{1}{2 \pi} \log \frac{|y|\left|x-\overline{y^{*}}\right|}{|y|\left|x-y^{*}\right|}+\frac{1}{2 \pi} \log \frac{|y|\left|x-\underline{y^{*}}\right|}{|y|\left|x-\overline{y^{*}}\right|} .
$$

Therefore if we set $\beta_{1}:=|y|^{2}\left|x-\underline{y^{*}}\right|^{2}$ and $\beta_{2}:=|y|^{2}\left|x-\underline{y^{*}}\right|^{2}$, then $\beta_{1}=\beta_{2}-4 x_{2} y_{2}$, hence $\log \beta_{1} / \beta_{2}<0$. This implies that

$$
\begin{aligned}
0 \leqslant \widehat{h}_{1}(x, y) \leqslant \frac{1}{2 \pi} \log \frac{|y|\left|x-\overline{y^{*}}\right|}{|y|\left|x-y^{*}\right|} & =\frac{1}{4 \pi} \log \left(1+\frac{4 x_{2} y_{2}}{|y|^{2}\left|x-y^{*}\right|^{2}}\right) \\
& \leqslant \frac{x_{2} y_{2}}{\pi(|x||y|-1)^{2}},
\end{aligned}
$$

provided $x, y \in \Pi_{+} \backslash \bar{B}_{1}$. Similarly, we obtain

$$
0 \leqslant \widehat{h}_{1}(x, y) \leqslant \frac{x_{1} y_{1}}{\pi(|x||y|-1)^{2}}
$$

provided $x, y \in \Pi_{+} \backslash \bar{B}_{1}$.

Let $\zeta \in L^{p}(\Omega)$ have compact support. Then $K_{+} \zeta(x)$ is defined at every point $x \in \mathbb{R}^{2}$, see [7]. Thus from

$$
|K \zeta(x)| \leqslant K|\zeta|(x) \leqslant K_{+}|\zeta|(x)
$$

it follows that $K \zeta(x)$ is defined at every $x \in \mathbb{R}$. Our first lemma is a standard result, see for example [6, Lemma 3.3].

LEMMA 1. Let $q \in[1, \infty)$ and let $U$ be an open, bounded subset of $\Omega$. Then $K: L^{p}(U) \rightarrow L^{q}(U)$ is compact. Moreover, if $\zeta \in L^{p}(\Omega)$ vanishes outside $U$, then

(i) $-\Delta K \zeta=\zeta$, almost everywhere in $\Omega$

(ii) $K \zeta=0$, on $\partial \Omega$

(iii) $K \zeta \in W_{\text {loc }}^{2, p}(\bar{\Omega})$, that is, for every open and bounded set $O \subset \Omega$ with $\bar{O} \subset \bar{\Omega}$ we have $K \zeta \in W^{2, p}(O)$. 
LEMma 2. Let $\zeta \in L^{p}(\Omega)$ have compact support. Then $K \zeta(x)=O\left(|x|^{-1}\right)$, $\nabla K \zeta(x)=O\left(|x|^{-2}\right)$, as $|x| \rightarrow \infty$.

PROOF: Let us recall that

$$
|K \zeta(x)| \leqslant K_{+}|\zeta|(x)
$$

for every $x \in \mathbb{R}^{2}$. Since $K_{+}|\zeta|(x)=O\left(|x|^{-1}\right)$ as $|x| \rightarrow \infty$, see [7], we deduce that $K \zeta(x)=O\left(|x|^{-1}\right)$ as $|x| \rightarrow \infty$. Hence if $A>0$, then there exists $M_{1}>0$ such that for $|x|>M_{1}$ we have $|K \zeta(x)| \leqslant A|x|^{-1}$. Now let us consider a special extension of $K \zeta$, denoted $(K \zeta)_{e}$, which is defined by

$$
(K \zeta)_{e}=\left\{\begin{aligned}
K \zeta(x) & x \in \Omega \\
-K \zeta(\underline{x}) & x \in \Omega_{-} \\
-K \zeta(\bar{x}) & x \in \Omega^{-}
\end{aligned}\right.
$$

where $\Omega_{-}, \Omega^{-}$denote the reflection of $\Omega$ about the lines $x_{1}=0, x_{2}=0$, respectively. Let us note that $(K \zeta)_{e}$ is harmonic in $\bar{\Omega} \cup \Omega^{-} \cup \Omega_{-} \backslash B_{M_{2}}$, for some $M_{2}>0$. Now consider $x$ such that $|x|>M:=\max \left\{2,2 M_{1}, 2 M_{2}\right\}$, then by Harnack's inequality [8, Theorem 2.10, p. 23] we obtain

$$
\left|\nabla(K \zeta)_{e}(x)\right| \leqslant \frac{8}{|x|} \sup _{z \in B_{|x| / 2}(x)}\left|(K \zeta)_{e}(z)\right| \leqslant 8 A|x|^{-2} .
$$

Hence we are done.

The next lemma is a result of Lemma 2 and the method for proving [7, Lemma 4].

LEMMA 3. Let $q$ and $U$ be as in Lemma 1. Then $K: L^{p}(U) \rightarrow L^{q}(U)$ is strictly positive, that is, for every non-trivial $\zeta \in L^{p}(\Omega)$, vanishing outside $U$,

$$
\int_{\Omega} \zeta K \zeta>0
$$

The following lemma has been proved in [7].

LEMmA 4. Let $\lambda>0$. Then there exists $R(\lambda)>0$ such that

$$
K_{+} \zeta(x)-\lambda x_{1} x_{2} \leqslant 0, \zeta \in \mathcal{F},|x|>R(\lambda)
$$

In fact $R(\lambda)=M / \lambda$, where $M$ is a positive constant.

REMARK. It is clear that the results of Lemmas 1-4 still hold if we replace $K$ by $K_{c}$.

The next lemma has been proved in [4].

LEMma 5. Let $(X, \mathcal{M}, \mu)$ be a finite separable nonatomic measure space, let $1 \leqslant p \leqslant \infty$, let $p^{*}$ be the conjugate exponent of $p$, let $\Psi: L^{p}(X) \rightarrow \mathbb{R}$ be convex, let $f_{0} \in L^{p}(X)$ and let $\mathcal{F}$ denote the set of rearrangements of $f_{0}$ on $X$. 
(i) Suppose that $\Psi$ is weakly sequentially continuous on $L^{p}(X)$. Then $\Psi$ attains a maximum value relative to $\mathcal{F}$.

(ii) Suppose $\Psi$ is strictly convex, that $f^{*}$ is a maximiser for $\Psi$ relative to $\mathcal{F}$ and that $g \in \partial \Psi\left(f^{*}\right)$, the subdifferential of $\Psi$ at $f^{*}$. Then $f^{*}=\phi \circ g$ almost everywhere in $X$ for some increasing $\phi$.

The following result is elementary.

Lemma 6. Let $c>0$, let $\mathcal{C}: \mathcal{F} \rightarrow \mathcal{F}_{c}$ be defined as in (4). Then $\mathcal{C}$ is a bijection. Moreover, if $\zeta \in \mathcal{F}$, then

(i) $\|\mathcal{C}(\zeta)\|_{p}=c^{1 / p^{*}}\|\zeta\|_{p}$

(ii) $|\operatorname{supp}(\mathcal{C}(\zeta))|=c^{-1}|\operatorname{supp}(\zeta)|$.

In our analysis we make use of the so-called Routh function $H(x)=H_{1}(x)+H_{2}(x)$, $x \in \Pi_{+}$, where

$$
\begin{aligned}
& H_{1}(x)=\frac{1}{4 \pi} \log \frac{|x|}{2 x_{1} x_{2}}, \\
& H_{2}(x)=x_{1} x_{2} .
\end{aligned}
$$

Observe that for $z \in \partial \Pi_{+}$we have $\lim _{x \rightarrow z} H(x)=\infty$. Elementary calculations prove that $H$ has a unique global minimum at $x_{0}=(1 /(2 \sqrt{2 \pi})),(1 /(2 \sqrt{2 \pi}))$. Occasionally the first and the second coordinates of $x_{0}$ are denoted by $x_{0,1}$ and $x_{0,2}$.

For more information on the Routh function the reader is referred to the classic monograph [10].

LEMMA 7 . Let $c$ and $\xi$ be positive constants satisfying (6). Let $\check{\zeta}_{c, \xi} \in \Sigma_{c, \xi}$; then

$$
\widehat{\Psi}_{c}\left(\check{\zeta}_{c, \xi}\right) \geqslant \frac{1}{4 \pi} \log \frac{c^{1 / 2}}{2 a}-C_{1}
$$

where $C_{1}$ is a positive constant, provided $c$ is sufficiently large.

Proof: Let $\zeta_{c, \xi}^{*}$ denote the Schwarz symmetrisation of $\check{\zeta}_{c, \xi}$ with respect to $x_{0}$. By Lemma 6 (ii) we have $\operatorname{supp}\left(\zeta_{c, \xi}^{*}\right)=B_{a / c^{1 / 2}}\left(x_{0}\right)$, hence, for sufficiently large $c$, we can ensure $\operatorname{supp}\left(\zeta_{c, \xi}^{*}\right) \subset \Omega_{c, \xi}$. Thus $\widehat{\Psi}_{c}\left(\check{\zeta}_{c, \xi}\right) \geqslant \widehat{\Psi}_{c}\left(\zeta_{c, \xi}^{*}\right)$. We now proceed to estimate $\widehat{\Psi}_{c}\left(\zeta_{c, \xi}^{*}\right)$ from below. From the definition of $\widehat{\Psi}_{c}$ we have

$$
\begin{aligned}
\widehat{\Psi}_{c}\left(\zeta_{c, \xi}^{*}\right)= & \frac{1}{2} \int_{\Omega_{c}} \zeta_{c, \xi}^{*} K_{c} \zeta_{c, \xi}^{*}-\int_{\Omega_{c}} \eta_{c} \zeta_{c, \xi}^{*} \\
= & \int_{\Omega_{c}} \int_{\Omega_{c}}\left(\frac{1}{4 \pi} \log \frac{1}{c^{1 / 2}|x-y|}-\frac{1}{2} h\left(c^{1 / 2} x, c^{1 / 2} y\right)\right) \zeta_{c, \xi}^{*}(x) \zeta_{c, \xi}^{*}(y) d x d y \\
& \quad-\int_{\Omega_{c}} \eta_{c}(x) \zeta_{c, \xi}^{*}(x) d x=I_{1}-I_{2}
\end{aligned}
$$

We now estimate $I_{1}$ as follows

$$
\begin{array}{r}
I_{1} \geqslant \frac{1}{4 \pi} \log \frac{1}{2 a}-\int_{\Omega_{c}} \int_{\Omega_{c}}\left(\frac{1}{2} h\left(c^{1 / 2} x, c^{1 / 2} y\right)-H_{1}\left(c^{1 / 2} x_{0}\right)\right) \zeta_{c, \xi}^{*}(x) \zeta_{c, \xi}^{*}(y) d x d y \\
-H_{1}\left(c^{1 / 2} x_{0}\right)
\end{array}
$$


where we have used $\left\|\zeta_{c, \xi}^{*}\right\|_{1}=1$. Now we show the integral in (12), denoted by $J_{1}$, is $o(1)$ as $c \rightarrow \infty$. First note that for $x, y \in \operatorname{supp}\left(\zeta_{c, \xi}^{*}\right)$ we have

$$
\left|\frac{1}{2} h\left(c^{1 / 2} x, c^{1 / 2} y\right)-H_{1}\left(c^{1 / 2} x_{0}\right)\right| \leqslant \frac{1}{2} \widehat{h}_{1}\left(c^{1 / 2} x, c^{1 / 2} y\right)+\left|\frac{1}{2} h_{+}\left(c^{1 / 2} x, c^{1 / 2} y\right)-H_{1}\left(c^{1 / 2} x_{0}\right)\right| .
$$

Now by applying (9) we deduce that

$$
\left|\frac{1}{2} h\left(c^{1 / 2} x, c^{1 / 2} y\right)-H_{1}\left(c^{1 / 2} x_{0}\right)\right| \leqslant \frac{c x_{2} y_{2}}{2 \pi(c|x||y|-1)^{2}}+\left|\frac{1}{2} h_{+}\left(c^{1 / 2} x, c^{1 / 2} y\right)-H_{1}\left(c^{1 / 2} x_{0}\right)\right|,
$$

for sufficiently large $c$. Next observe that

$$
\sup _{x, y \in \operatorname{supp}\left(\zeta_{c, \xi}^{*}\right)} \frac{c x_{2} y_{2}}{2 \pi(c|x||y|-1)^{2}} \rightarrow 0
$$

as $c \rightarrow \infty$; also

$\sup _{x, y \in \operatorname{supp}\left(\zeta_{c, \xi}^{*}\right)}\left|\frac{1}{2} h_{0}\left(c^{1 / 2} x, c^{1 / 2} y\right)-H_{1}\left(c^{1 / 2} x_{0}\right)\right|=\sup _{x, y \in \operatorname{supp}\left(\zeta_{c, \xi}^{*}\right)}\left|\frac{1}{4 \pi} \log \frac{2|x-\bar{y}| x_{0,1} x_{0,2}}{|x-\bar{y}||x-\underline{y}|\left|x_{0}\right|}\right| \rightarrow 0$

as $c \rightarrow \infty$, where

$$
h_{0}(x, y)=\frac{1}{2 \pi} \log \frac{|x-\underline{y}|}{|x-\bar{y}||x-\underline{y}|} .
$$

Therefore $J_{1}=o(1)$ as $c \rightarrow \infty$, whence

$$
I_{1} \geqslant \frac{1}{4 \pi} \log \frac{1}{2 a}-H_{1}\left(c^{1 / 2} x_{0}\right)-o(1),
$$

as $c \rightarrow \infty$.

Now we estimate $I_{2}$,

$$
\begin{aligned}
I_{2} & =\int_{\Omega_{c}} \eta_{c}(x) \zeta_{c, \xi}^{*}(x) d x \\
& =\int_{\Omega_{c}}\left(\eta_{c}(x)-x_{0,1} x_{0,2}\right) \zeta_{c, \xi}^{*}(x) d x+H_{2}\left(x_{0}\right) .
\end{aligned}
$$

Note that from (3) we infer that

$$
x_{1} x_{2}-\frac{x_{1} x_{2}}{c^{2}|x|^{4}} \leqslant \eta_{c}(x) \leqslant x_{1} x_{2}, \quad x \in \Omega_{c} .
$$

Hence

$$
\sup _{x, y \in \operatorname{supp}\left(\zeta_{c, \xi}^{*}\right)}\left|\eta_{c}(x)-x_{0,1} x_{0,2}\right| \leqslant \sup _{x, y \in \operatorname{supp}\left(\zeta_{c, \xi}\right)}\left(2\left|x_{1} x_{2}-x_{0,1} x_{0,2}\right|+1 /\left(c^{2}|x|^{2}\right)\right) \rightarrow 0,
$$

as $c \rightarrow \infty$. Therefore

$$
I_{2}=H_{2}\left(x_{0}\right)+o(1),
$$


as $c \rightarrow \infty$. Now from (13) and (14) we deduce that

$$
\widehat{\Psi}_{c}\left(\zeta_{c, \xi}^{*}\right) \geqslant \frac{1}{4 \pi} \log \frac{1}{2 a}-H_{1}\left(c^{1 / 2} x_{0}\right)-H_{2}\left(x_{0}\right)+o(1)
$$

as $c \rightarrow \infty$, or

$$
\widehat{\Psi}_{c}\left(\zeta_{c, \xi}^{*}\right) \geqslant \frac{1}{4 \pi} \log \frac{c^{1 / 2}}{2 a}-H\left(x_{0}\right)+o(1)
$$

as $c \rightarrow \infty$. This clearly verifies (11).

Now assume $c$ and $\xi$ satisfy (6). From Lemma 1 it follows that $\widehat{\Psi}_{c}: L^{p}\left(\Omega_{c, \xi}\right) \rightarrow \mathbb{R}$ is weakly sequentially continuous. Moreover, from Lemma $3, \widehat{\Psi}_{c}$ is also strictly positive. Thus problem $\widehat{P}_{c, \xi}$ is solvable by Lemma 5 (i). Since $\widehat{\Psi}_{c}$ is differentiable, the subdifferential of $\widehat{\Psi}_{c}$ at say $\zeta$ is a singleton, namely the derivative of $\widehat{\Psi}_{c}$ at $\zeta$, which is identified with $K_{c} \zeta-\eta_{c}$. Therefore if $\check{\zeta}_{c, \xi} \in \Sigma_{c, \xi}$, then by Lemma 5 (ii) there exists an increasing function $\phi_{c, \xi}$ such that

$$
\check{\zeta}_{c, \xi}=\phi_{c, \xi} \circ\left(K_{c} \check{\zeta}_{c, \xi}-\eta_{c}\right) \text { almost everywhere in } \Omega_{c, \xi}
$$

From this it follows

$$
\operatorname{supp}\left(\check{\zeta}_{c, \xi}\right)=\left\{x \in \Omega_{c, \xi} \mid K_{c} \check{\zeta}_{c, \xi}(x)-\eta_{c}(x) \geqslant \gamma_{c, \xi}\right\},
$$

for some constant $\gamma_{c, \xi}$, modulo a set of measure zero. Note that the inequality in (15) can be changed to strict inequality, since the level sets of $K_{c} \check{\zeta}_{c, \xi}-\eta_{c}$ (sets on which $K_{c} \check{\zeta}_{c, \xi}-\eta_{c}$ is constant) on $\operatorname{supp}\left(\check{\zeta}_{c, \xi}\right)$ have measure zero, by [8, Lemma 7.7]. In the next lemma we derive a lower bound for $\gamma_{c, \xi}$ when $c$ and $\xi$ are sufficiently large.

LEMmA 8. There exists $c_{1}>0$ and $\xi_{1}>0$ such that if $c \geqslant c_{1}$ and $\xi \geqslant \xi_{1}$, then

$$
\gamma_{c, \xi} \geqslant \frac{1}{2 \pi} \log \frac{c^{1 / 2}}{2 a}+C_{2}(k)
$$

where $C_{2}(k)$ is a constant depending on $k$, the constant depending on the cone determining the cone property of $\Omega_{c, \xi}$.

PROOF: Let $c_{1}^{\prime}$ and $\xi_{1}^{\prime}$ be positive constants such that if $c \geqslant c_{1}^{\prime}$ and $\xi \geqslant \xi_{1}^{\prime}$ satisfy (6), then $B \equiv B_{1 /(2 \sqrt{2 \pi})}\left(x_{0}\right) \subset \Omega_{c, \xi}$. Let $\tilde{\gamma}>0$ be such that $B \subset \Pi_{+}(\tilde{\gamma}):=\left\{x \in \Pi_{+} \mid\right.$ $\left.x_{1} x_{2}<\tilde{\gamma}\right\}$. We claim that by merely increasing $c$ we can ensure $\gamma_{c, \xi} \geqslant-\widetilde{\gamma}$. To seek a contradiction suppose $\gamma_{c, \xi}<-\widetilde{\gamma}$; then for $x \in B$ we have

$$
K_{c} \check{\zeta}_{c, \xi}(x)-\eta_{c}(x) \geqslant-\eta_{c}(x) \geqslant-x_{1} x_{2}>-\tilde{\gamma}>\gamma_{c, \xi},
$$

since $K_{c} \check{\zeta}_{c, \xi}$ is non-negative and $\eta_{c} \leqslant x_{1} x_{2}$. Therefore $B \subset \operatorname{supp}\left(\check{\zeta}_{c, \xi}\right)$, modulo a set of measure zero. Hence $|B| \leqslant\left|\operatorname{supp}\left(\check{\zeta}_{c, \xi}\right)\right|$, that is, $1 / 8 \leqslant \pi a^{2} / c$, so $c \leqslant 8 \pi a^{2}$. To derive a contradiction it suffices to make $c$ greater than $8 \pi a^{2}$. Henceforth we assume $c \geqslant \max \left\{c_{1}^{\prime}, 8 \pi a^{2}\right\}$. Therefore we obtain

$$
\operatorname{supp}\left(\check{\zeta}_{c, \xi}\right) \subseteq\left\{x \in \Omega_{c, \xi} \mid K_{c} \check{\zeta}_{c, \xi}(x)-\eta_{c}(x)>-\tilde{\gamma}\right\}
$$


modulo a set of measure zero. Let us now define the adjusted energy functional

$$
F(\zeta):=\frac{1}{2} \int_{\Omega_{c}}\left(K_{c} \zeta-\eta_{c}-\gamma_{c, \xi}\right) \zeta
$$

for measurable functions $\zeta$ on $\Omega_{c}$. Observe that

$$
F\left(\check{\zeta}_{c, \xi}\right) \leqslant \frac{1}{2} \int_{\Omega_{c}} u^{+} \check{\zeta}_{c, \xi}-\frac{1}{2}\left(\gamma_{0}-1\right)
$$

where $\gamma_{0}:=-\tilde{\gamma}$ and $u:=K_{c} \check{\zeta}_{c, \xi}-\eta_{c}-\gamma_{c, \xi}+\gamma_{0}-1$. It is clear that there exists $M>\xi$ such that $u^{+} \in H_{0}^{1}\left(\Omega_{c, M}\right)$. Now by applying the "half Green formula", see [9, p. 24], and Lemma 1 (ii), applied to $K_{c} \check{\zeta}_{c, \xi}$ we find

$$
\left\|\nabla u^{+}\right\|_{2, \Omega_{c, \xi}}^{2} \leqslant\left\|\nabla u^{+}\right\|_{2, \Omega_{c, M}}^{2}=\int_{\Omega_{c, M}} \nabla u^{+} \cdot \nabla u^{+}=\int_{\Omega_{c, M}} \nabla u^{+} \cdot \nabla u=-\int_{\Omega_{c, \xi}} u^{+} \check{\zeta}_{c, \xi}
$$

Hence we can apply Hölder's inequality to obtain

$$
\left\|\nabla u^{+}\right\|_{2, \Omega_{c, \xi}}^{2} \leqslant\left\|u^{+}\right\|_{2, \Omega_{c, \xi}}\left\|\check{\zeta}_{c, \xi}\right\|_{2, \Omega_{c, \xi}}
$$

where we have used Lemma 6(i). From the continuous embedding

$$
W^{1,1}\left(\Omega_{c, \xi}\right) \hookrightarrow L^{2}\left(\Omega_{c, \xi}\right)
$$

see $[1$, p. 105$]$, we deduce that

$$
\left\|u^{+}\right\|_{2, \Omega_{c, \xi}} \leqslant k\left\|u^{+}\right\|_{1,1, \Omega_{c, \xi}}
$$

where $k$ is the constant depending on the cone determining the cone property of $\Omega_{c, \xi}$; let us point out that the cone is independent of $c$ and $\xi$, hence, in turn, $k$ is independent of $c$ and $\xi$. Next we observe that

$$
\left\|u^{+}\right\|_{1,1, \Omega_{c, \xi}} \leqslant\left\|u^{+}\right\|_{2, \Omega_{c, \xi}}+2\left\|\nabla u^{+}\right\|_{1, \Omega_{c, \xi}}
$$

Note that $\operatorname{supp}\left(u^{+}\right)$and $\operatorname{supp}\left(\nabla u^{+}\right)$are both contained in $\operatorname{supp}(u)$; and since $\operatorname{supp}(u)$ is essentially contained in $\operatorname{supp}\left(\check{\zeta}_{c, \xi}\right)$ we deduce that $\operatorname{supp}\left(u^{+}\right)$and $\operatorname{supp}\left(\nabla u^{+}\right)$are essentially contained in $\operatorname{supp}\left(\check{\zeta}_{c, \xi}\right)$. This implies that

$$
\left\|u^{+}\right\|_{1, \Omega_{c, \xi}}=\left\|u^{+}\right\|_{1, \operatorname{supp}\left(\bar{\zeta}_{c, \xi}\right)} \leqslant\left|\operatorname{supp}\left(\check{\zeta}_{c, \xi}\right)\right|^{1 / 2}\left\|u^{+}\right\|_{2, \Omega_{c, \xi}}=\frac{\sqrt{\pi} a}{c^{1 / 2}}\left\|u^{+}\right\|_{2, \Omega_{c, \xi}}
$$

where we have used Hölder's inequality and Lemma 6 (ii). Similarly we obtain

$$
\left\|\nabla u^{+}\right\|_{1, \Omega_{c, \xi}} \leqslant \frac{\sqrt{\pi} a}{c^{1 / 2}}\left\|\nabla u^{+}\right\|_{2, \Omega_{c, \xi}}
$$

Therefore we derive

$$
\left\|u^{+}\right\|_{2, \Omega_{c, \xi}} \leqslant k\left(\frac{\sqrt{\pi} a}{c^{1 / 2}}\left\|u^{+}\right\|_{2, \Omega_{c, \xi}}+\frac{2 \sqrt{\pi} a}{c^{1 / 2}}\left\|\nabla u^{+}\right\|_{2, \Omega_{c, \xi}}\right) .
$$


This, in turn, implies that

$$
\left(1-\frac{k \sqrt{\pi} a}{c^{1 / 2}}\right)\left\|u^{+}\right\|_{2, \Omega_{c, \xi}} \leqslant \frac{2 k \sqrt{\pi} a}{c^{1 / 2}}\left\|\nabla u^{+}\right\|_{2, \Omega_{c, \xi}} .
$$

Let $c_{1}^{\prime \prime}$ be a positive number such that $c \geqslant c_{1}^{\prime \prime}$ implies $\left(1-k \sqrt{\pi} a / c^{1 / 2}\right)>1 / 2$, then for $c \geqslant \max \left\{c_{1}^{\prime}, c_{1}^{\prime \prime}, 8 \pi a^{2}\right\}$ we obtain

$$
\frac{1}{2}\left\|u^{+}\right\|_{2, \Omega_{c, \xi}} \leqslant \frac{2 k \sqrt{\pi} a}{c^{1 / 2}}\left\|\nabla u^{+}\right\|_{2, \Omega_{c, \xi}}
$$

From (19) and (20) we deduce that

$$
\left\|\nabla u^{+}\right\|_{2, \Omega_{c, \xi}}^{2} \leqslant 4 k \sqrt{\pi} a\left\|\zeta_{0}\right\|_{2}\left\|\nabla u^{+}\right\|_{2, \Omega_{c, \xi}}
$$

hence

$$
\left\|\nabla u^{+}\right\|_{2, \Omega_{c, \xi}} \leqslant 4 k \sqrt{\pi} a\left\|\zeta_{0}\right\|_{2}
$$

Therefore by applying Hölder's inequality, (20) and (21) we obtain

$$
\begin{aligned}
\int_{\Omega_{c, \xi}} u^{+} \check{\zeta}_{c, \xi} \leqslant c^{1 / 2}\left\|\zeta_{0}\right\|_{2}\left\|u^{+}\right\|_{2, \Omega_{c, \xi}} & \leqslant 4 k \sqrt{\pi} a\left\|\zeta_{0}\right\|_{2}\left\|\nabla u^{+}\right\|_{2, \Omega_{c, \xi}} \\
& \leqslant\left(4 k \sqrt{\pi} a\left\|\zeta_{0}\right\|_{2}\right)^{2}=: \widetilde{\beta}(k) .
\end{aligned}
$$

From this we infer that

$$
F\left(\check{\zeta}_{c, \xi}\right) \leqslant \frac{1}{2}\left(\widetilde{\beta}(k)-\gamma_{0}+1\right)=: \beta(k) .
$$

From (18) we also have

$$
\widehat{\Psi}_{c}\left(\check{\zeta}_{c, \xi}\right)=F\left(\check{\zeta}_{c, \xi}\right)-\frac{1}{2} \int_{\Omega_{c, \xi}} \eta_{c} \check{\zeta}_{c, \xi}+\frac{1}{2} \gamma_{c, \xi} \leqslant F\left(\check{\zeta}_{c, \xi}\right)+\frac{1}{2} \gamma_{c, \xi}
$$

By Lemma 7 there exists $c_{1}^{\prime \prime \prime}>0$ such that for $c \geqslant c_{1}^{\prime \prime \prime}$ we have

$$
\widehat{\Psi}_{c}\left(\check{\zeta}_{c, \xi}\right) \geqslant \frac{1}{4 \pi} \log \frac{c^{1 / 2}}{2 a}-C_{1} .
$$

Hence if $c \geqslant \max \left\{c_{1}^{\prime}, c_{1}^{\prime \prime}, c_{1}^{\prime \prime \prime}, 8 \pi a^{2}\right\}$, then by (22) we have

$$
F\left(\check{\zeta}_{c, \xi}\right)+\frac{1}{2} \gamma_{c, \xi} \geqslant \frac{1}{4 \pi} \log \frac{c^{1 / 2}}{2 a}-C_{1}
$$

Since $F\left(\check{\zeta}_{c, \xi}\right) \leqslant \beta(k)$ we finally obtain

$$
\gamma_{c, \xi} \geqslant \frac{1}{2 \pi} \log \frac{c^{1 / 2}}{2 a}-2\left(C_{1}+\beta(k)\right) \text {. }
$$

This readily implies (16), for $c_{1}:=\max \left\{c_{1}^{\prime}, c_{1}^{\prime \prime}, c_{1}^{\prime \prime}, 8 \pi a^{2}\right\}$ and $\xi_{1}:=\xi_{1}^{\prime}$. 


\section{Proof of THE.THEOREM}

Let $c_{1}$ and $\xi_{1}$ be as in Lemma 8. Let $c_{2}$ be a positive constant such that for $c \geqslant c_{2}$,

$$
\frac{1}{2 \pi} \log \frac{c^{1 / 2}}{2 a}-C_{2}(k)>1 \text {. }
$$

Let us consider $c \geqslant c_{0}=\max \left\{1, c_{1}, c_{2}\right\}, \xi \geqslant \xi_{1}$ and $\check{\zeta}_{c, \xi} \in \Sigma_{c, \xi}$, this set is not empty by Lemma 5. From (15), (23), and Lemma 8 we obtain

$$
\operatorname{supp}\left(\check{\zeta}_{c, \xi}\right) \subset \operatorname{supp}\left(K_{c} \check{\zeta}_{c, \xi}-\eta_{c}\right)
$$

modulo a set of measure zero. Let us observe that for $x \in \Omega_{c}$ we have

$$
K_{c} \check{\zeta}_{c, \xi}(x)=K \mathcal{C}^{-1}\left(\check{\zeta}_{c, \xi}\right)\left(c^{1 / 2} x\right)
$$

Moreover, since $K \mathcal{C}^{-1}\left(\check{\zeta}_{c, \xi}\right)\left(c^{1 / 2} x\right) \leqslant K_{+} \mathcal{C}^{-1}\left(\check{\zeta}_{c, \xi}\right)\left(c^{1 / 2} x\right)$ we infer that

$$
K_{c} \check{\zeta}_{c, \xi}(x) \leqslant K_{+} \mathcal{C}^{-1}\left(\check{\zeta}_{c, \xi}\right)\left(c^{1 / 2} x\right),
$$

for $x \in \Omega_{c}$. Also, if $x \in \Omega_{c}$ is such that $|x|>1$, then by applying (3) we obtain

$$
\eta_{c}(x) \geqslant \frac{1}{2} x_{1} x_{2}
$$

hence, for $x \in \Omega_{c}$ such that $|x|>1$, we derive that

$$
\begin{aligned}
K_{c} \check{\zeta}_{c, \xi}(x)-\eta_{c}(x) & =K \mathcal{C}^{-1}\left(\check{\zeta}_{c, \xi}\right)\left(c^{1 / 2} x\right)-\eta_{c}(x) \\
& \leqslant K_{+} \mathcal{C}^{-1}\left(\check{\zeta}_{c, \xi}\right)\left(c^{1 / 2} x\right)-1 / 2 x_{1} x_{2}
\end{aligned}
$$

By Lemma 4 we have

$$
K_{+} \mathcal{C}^{-1}\left(\check{\zeta}_{c, \xi}\right)\left(c^{1 / 2} x\right)-1 / 2 x_{1} x_{2} \leqslant 0,
$$

provided $|x| \geqslant A c^{1 / 2}$, where $A$ is some universal positive constant. Let us now define $R(c):=\max \left\{1, \xi_{1}, A c^{1 / 2}\right\}$. Then from (24) we obtain

$$
\operatorname{supp}\left(\check{\zeta}_{c, \xi}\right) \subset B_{R(c)}
$$

Fixing $c$, clearly, (25) holds for every $\xi \geqslant \xi_{1}$. Hence $\check{\zeta}_{c, R(c)} \in \Sigma_{c}$. This concludes the existence part of the theorem.

We now proceed to derive (7). Henceforth we assume $c$ is a fixed number such that $c \geqslant c_{0}$. Consider $\check{\zeta}_{c} \in \Sigma_{c}$. Hence $\widehat{\zeta}_{\lambda}:=\mathcal{C}^{-1}\left(\check{\zeta}_{c}\right) \in \Sigma_{\lambda}$, where $\lambda=1 / c$. It is clear that $\operatorname{supp}\left(\widehat{\zeta}_{\lambda}\right) \subset B_{c^{1 / 2} R(c)}$, modulo a set of zero measure. Applying Lemma 8 and (23) we find

$$
K_{c} \check{\zeta}_{c}(x)-\eta_{c}(x) \geqslant 1
$$

for almost every $x \in \operatorname{supp}\left(\check{\zeta}_{c}\right)$, or equivalently

$$
K \mathcal{C}^{-1}\left(\check{\zeta}_{c}\right)\left(c^{1 / 2} x\right)-\frac{1}{c} \eta\left(c^{1 / 2} x\right) \geqslant 1,
$$


for almost every $x \in \operatorname{supp}\left(\check{\zeta}_{c}\right)$, whence $K \widehat{\zeta}_{\lambda}(x)-\lambda \eta(x) \geqslant 1$, for almost every $x \in \operatorname{supp}\left(\widehat{\zeta}_{\lambda}\right)$. Let us now observe that $\limsup _{|x| \rightarrow \infty}\left(K \widehat{\zeta}_{\lambda}(x)-\lambda \eta(x)\right) \leqslant 0$. Hence there exists $M_{3} \geqslant c^{1 / 2} R(c)$ such that $\left.K \widehat{\zeta}_{\lambda}(x)-\lambda \eta(x)\right) \leqslant 1 / 2$, provided $|x|>M_{3}$. Since $\widehat{\zeta}_{\lambda}$ is a global maximiser of $\Psi_{\lambda}$ relative to $\mathcal{F}$ we deduce that, in particular, $\widehat{\zeta}_{\lambda}$ maximises $\Psi_{\lambda}$ relative to functions in $\mathcal{F}$ which vanish outside $\Omega_{M_{3}}:=B_{M_{3}} \cap \Omega$. Therefore, by Lemma 5 , there exists an increasing function $\phi$ such that

$$
\widehat{\zeta}_{\lambda}=\phi \circ\left(K \widehat{\zeta}_{\lambda}-\lambda \eta\right)
$$

for almost every $x \in \Omega_{M_{3}}$. We modify $\phi$ by $\widehat{\phi}_{\lambda}$ which is defined as follows

$$
\widehat{\phi}_{\lambda}(s):=\left\{\begin{array}{cc}
\phi_{\lambda}(s), & s \geqslant 1 \\
0, & s<1 .
\end{array}\right.
$$

Therefore we derive

$$
\widehat{\zeta}_{\lambda}=\widehat{\phi}_{\lambda} \circ\left(K \widehat{\zeta}_{\lambda}-\lambda \eta\right)
$$

for almost every $x \in \Omega$. Since $\widehat{\zeta}_{\lambda}=-\Delta K \widehat{\zeta}_{\lambda}$, for almost everywhere $x \in \Omega$, we deduce (7). Note that $\lambda_{0}:=1 / c_{0}$.

\section{REFERENCES}

[1] R.A. Adams, Sobolev spaces, Pure and Applied Maths. 65 (Academic Press, New York, London, 1975).

[2] T.V. Badiani, 'Existence of steady symmetric vortex pairs on a planar domain with an obstacle', Math. Proc. Cambridge Philos. Soc. 123 (1998), 335-384.

[3] T.B. Benjamin, 'The alliance of practical and analytical insights into the nonlinear problems of fluid mechanics', in Applications of methods of functional analysis to problems in mechanics, Lecture Notes in Mathematics 503 (Springer-Verlag, Berlin, 1976), pp. 8-29.

[4] G.R. Burton, 'Rearrangements of functions, maximisation of convex functionals and vortex rings', Math. Ann. 276 (1987), 225-253.

[5] G.R. Burton and B. Emamizadeh, 'A constrained variational problem for steady vortices in a shear flow', Comm. Partial Differential Equations 24 (1999), 1341-1365.

[6] B. Emamizadeh, 'Steady vortex in a uniform shear flow of an ideal fluid', Proc. Roy. Soc. Edinburgh Sect. A 130 (2000), 801-812.

[7] B. Emamizadeh, 'Existence of a steady flow with a bounded vortex in an unbounded domain', J. Sciences Islam. Repub. Iran 12 (2001), 57-63.

[8] D. Gilbarg, N.S. Trudinger, Elliptic partial differential equations of second order, (Second edition) (Springer-Verlag, Berlin, New York, 1977).

[9] P. Grisvard, Singularities in boundary value problems, Research in Applied Mathematics 22 (Springer-Verlag, Berlin, 1992).

[10] C.C. Lin, On the notions of vortices in two dimensions (Univ. of Toronto Press, Toronto, 1943). 
[11] B. Turkington, 'On steady vortex flow in two dimensions, I', Comm. Partial Differential Equations 8 (1983), 999-1030.

[12] B. Turkington, 'On steady vortex flow in two dimensions, II', Comm. Partial Differential Equations 8 (1983), 1031-1071.

Department of Mathematics

Iran University of Science and Technology

Narmak 16844

Tehran

Iran

and

Institute for Studies in Theoretical Physics and Mathematics

Niavaran square

Tehran

Iran
Department of Mathematics

University of Tarbiat Modarres

Tehran

Iran 